\title{
Participation of Sodium Currents in Burst Generation and Control of Membrane Excitability in Mesencephalic Trigeminal Neurons
}

\author{
Akifumi Enomoto, ${ }^{1,2}$ Juliette M. Han, ${ }^{1}$ Chie-Fang Hsiao, ${ }^{1}$ Nanping Wu, ${ }^{1}$ and Scott H. Chandler ${ }^{1}$ \\ ${ }^{1}$ Department of Physiological Science, University of California at Los Angeles, Los Angeles, California 90095, and ${ }^{2}$ First Department of Oral Maxillofacial \\ Surgery, Graduate School of Dentistry, Osaka University, Osaka 565-0871, Japan
}

\begin{abstract}
Subthreshold sodium currents are important in sculpting neuronal discharge and have been implicated in production and/or maintenance of subthreshold membrane oscillations and burst generation in mesencephalic trigeminal neurons (Mes V). Moreover, recent data suggest that, in some CNS neurons, resurgent sodium currents contribute to production of high-frequency burst discharge. In the present study, we sought to determine more directly the participation of these currents during Mes $\mathrm{V}$ electrogenesis using the action potentialclamp method. In postnatal day 8-14 rats, the whole-cell patch-clamp method was used to record sodium currents by subtraction in response to application of TTX in voltage-clamp mode using the action potential waveform as the command protocol. We found that TTX-sensitive sodium current is the main inward current flowing during the interspike interval, compared with the h-current $\left(I_{\mathrm{h}}\right)$ and calcium currents. Furthermore, in addition to the transient sodium current that flows during the upstroke of action potential, we show that resurgent sodium current flows at the peak of afterhyperpolarization and persistent sodium current flows in the middle of the interspike interval to drive high-frequency firing. Additionally, transient, resurgent, and persistent sodium current components showed voltage- and time-dependent slow inactivation, suggesting that slow inactivation of these currents can contribute to burst termination. The data suggest an important role for these components of the sodium current in Mes V neuron electrogenesis.
\end{abstract}

Key words: subthreshold oscillations; resurgent sodium current; persistent sodium current; interspike interval; slow inactivation; oralmotor activity

\section{Introduction}

Historically, sodium channels are characterized as essential for action potential generation, yet it is now well known that there are not only transient sodium currents $\left(I_{\mathrm{NaT}}\right)$, but in many neurons, persistent, or slowly inactivating sodium currents $\left(I_{\mathrm{NaP}}\right)$ active within the subthreshold region (Chandler et al., 1994; Crill, 1996; Boehmer et al., 2000; Taddese and Bean, 2002; Do and Bean, 2003). More recently, a novel sodium current termed "resurgent sodium current" $\left(I_{\mathrm{NaR}}\right)$, which activates during spike repolarization, was described (Raman and Bean, 1997). This current would add additional inward current after an action potential and, therefore, contribute to spike frequency control (Raman and Bean, 1997). Each of these sodium current components, with their unique voltage- and time-dependent properties, potentially contributes to spike electrogenesis. The challenge is to understand how these different sodium currents are integrated to produce specific neuronal discharge patterns.

Mesencephalic trigeminal neurons (Mes V) are primary sensory neurons with cell bodies within the nervous system (Corbin

\footnotetext{
Received Dec. 9, 2005; revised Feb. 8, 2006; accepted Feb. 9, 2006.

This work was supported by National Institute of Dental and Craniofacial Research Grant DE 06193.

Correspondence should be addressed to Dr. Scott H. Chandler, Department of Physiological Science, University of California at Los Angeles, 2859 Slichter Hall, Los Angeles, CA 90095. E-mail: schandler@physci.ucla.edu. D0I:10.1523/JNEUROSC1.5274-05.2006

Copyright $\odot 2006$ Society for Neuroscience $\quad 0270-6474 / 06 / 263412-11 \$ 15.00 / 0$
}

and Harrison, 1940; Jerge, 1963). They relay sensory inputs from the jaw-closer muscle spindles and periodontal mechanoreceptors to trigeminal jaw-closer motoneurons, interneurons, thalamus, and other brainstem nuclei (Corbin and Harrison, 1940; Alvarado-Mallart et al., 1975; Appenteng et al., 1978, 1985; Dessem and Taylor, 1989). Additionally, there is evidence that Mes V neurons form coupled networks among themselves via electrical and excitatory recurrent connections (Baker and Llinas, 1971; Luo and Dessem, 1996). Although they are not directly responsible for setting the oral-motor rhythm during mastication or sucking, they are important for production of appropriate jaw movements (Miles, 1979; Morimoto et al., 1989) and could participate as interneurons because of their central brainstem location and synaptic connectivity (Kolta et al., 1995). As such, they are active during fictive mastication and local stimulation of nearby interneurons produces synaptic potentials, subthreshold oscillations, and spike activity in some Mes V neurons (Verdier et al., 2004).

Recently, it was demonstrated that Mes V neurons show conditional burst generation in response to maintained membrane depolarization (Pedroarena et al., 1999; Wu et al., 2001, 2005). Evidence was provided that $I_{\mathrm{NaP}}$ amplifies a $\mathrm{K}^{+}$-dependent membrane resonance to initiate subthreshold membrane oscillations. These subthreshold oscillations then give rise to burst generation (Wu et al., 2001, 2005). However, direct evidence for the 
precise role of this and other sodium current components $\left(I_{\mathrm{NaT}}\right.$ and $\left.I_{\mathrm{NaR}}\right)$ in maintaining high-frequency spike discharge during a burst, as well as in burst termination and subsequent burst initiation are not clear. In this study, we sought to address these issues with more direct electrical measurements of sodium current components using the action potential-clamp method, whereby the command voltage-clamp template is obtained from actual current-clamp waveforms during bursting and then used as a stimulus protocol during voltage clamp (Llinas et al., 1982; Do and Bean, 2003). We find that, in addition to a role for $I_{\mathrm{NaP}}$ during the interspike interval (ISI) in maintaining spike discharge, $I_{\mathrm{NaR}}$ is present immediately after spike repolarization and produces an additional inward current that likely contributes to the ability of these neurons to produce high-frequency discharge during a burst. Preliminary data have been presented (Enomoto et al., 2004).

\section{Materials and Methods}

Preparation of tissue. Experiments were performed on Mes V neurons. Coronal slices from neonatal Sprague Dawley rats (postnatal day 8-14) were cut by the protocol described previously (Wu et al., 2001, 2005). Briefly, animals were rapidly decapitated, and the brains were quickly removed and immersed in oxygenated $\left(95 \% \mathrm{O}_{2}-5 \% \mathrm{CO}_{2}\right)$ ice-cold cutting solution of the following composition (in mM): $126 \mathrm{NaCl}, 3 \mathrm{KCl}$, $1.25 \mathrm{NaH}_{2} \mathrm{PO}_{4}, 26 \mathrm{NaHCO}_{3}, 10$ glucose, $1 \mathrm{CaCl}_{2}, 5 \mathrm{MgCl}_{2}$, and 4 lactic acid (Schurr et al., 1988). The brainstem was glued by its rostral end to the platform of a chamber and covered with ice-cold cutting solution. Six slices $(300 \mu \mathrm{m})$ were cut on a vibrating slicer (DSK microslicer; Ted Pella, Redding, CA) and then placed at room temperature into an oxygenated incubation solution of the following composition (in mM): $124 \mathrm{NaCl}, 3$ $\mathrm{KCl}, 1.25 \mathrm{NaH}_{2} \mathrm{PO}_{4}, 26 \mathrm{NaHCO}_{3}, 10$ glucose, $2 \mathrm{CaCl}_{2}, 2 \mathrm{MgCl}_{2}$, and 4 lactic acid (Schurr et al., 1988). The slices were incubated at $37^{\circ} \mathrm{C}$ for $40-50 \mathrm{~min}$, and then maintained at room temperature $\left(22-24^{\circ} \mathrm{C}\right)$.

Electrophysiological technique. Patch electrodes were fabricated from borosilicate glass capillary tubing $(1.5 \mathrm{~mm}$ outer diameter; $0.86 \mathrm{~mm}$ inner diameter) using a model P-97 puller (Sutter Instrument, Novato, CA). The patch pipettes were wrapped near the tip with silicone agent (SYLGARD; Dow Corning, Midland, MI) to reduce capacitance. Wholecell current- and voltage-clamp recordings were performed with an Axopatch-1D amplifier (Molecular Devices, Foster City, CA) in concert with pCLAMP acquisition software (version 9.2; Molecular Devices). Signals were grounded ( $\mathrm{Ag} / \mathrm{AgCl}$ wire) using a $3 \mathrm{~m} \mathrm{KCl}$ agar bridge. After the establishment of a gigaohm seal, the whole-cell configuration was obtained by brief suction. Cells with seals $<1 \mathrm{G} \Omega$ before breakthrough were discarded. Uncompensated series resistance was usually $<10 \mathrm{M} \Omega$, compensated $60-90 \%$, and monitored periodically throughout the experiment. The data were low-pass filtered at $10 \mathrm{kHz}$ and sampled at $10-50 \mathrm{kHz}$ depending on the experiment. Liquid junction potentials were measured and corrected off-line (Zhang and Krnjevic, 1993). Electrode resistances were $3-5 \mathrm{M} \Omega$ when filled with the intracellular solution. Slices were secured in a recording chamber and perfused with oxygenated ACSF $(2-3 \mathrm{ml} / \mathrm{min})$ at room temperature and visualized by infrared differential interference contrast microscopy (Stuart et al., 1993). Additionally, in some experiments, outside-out patches were obtained and compared with voltage-clamp data of fast $I_{\mathrm{NaT}}$ in whole-cell configuration (see Fig. $7 D$ ). Steady-state $I-V$ plots were obtained and compared for both conditions. The overlap of the plots when scaled for amplitude suggested that, for whole-cell configuration, the space clamp was adequate to clamp the fast $I_{\mathrm{NaT}}$ (see Results).

The mesencephalic trigeminal nucleus was identified bilaterally in the coronal slice under low magnification $(5 \times)$ as an ellipsoid region, which is located dorsal and medial to the trigeminal motor nucleus, and $\sim 0.5$ $\mathrm{mm}$ lateral to the midline. Mesencephalic V neurons were easily distinguished based on their location, pseudo-unipolar soma, and size (Henderson et al., 1982; Del Negro and Chandler, 1997). The effects of drugs applied to the bath solution were obtained $3 \sim 10 \mathrm{~min}$ after application. Recording periods were usually between 30 and $60 \mathrm{~min}$.
Current- and voltage-clamp experiments. Burst discharge was elicited by maintained positive current injection under current-clamp mode, and the bursts were subsequently recorded ( $n=84$ neurons). To record underlying ionic currents responsible for burst discharge, a "typical" burst waveform template was used as the voltage command waveform during voltage-clamp experiments. Ideally, it is desirable to use the burst waveform associated with bursting from each neuron (Do and Bean 2003). However, in our experiments, this was not feasible, because the length of time it took to generate a voltage-clamp protocol from the recorded current-clamp waveform for each neuron was too long. Therefore, a typical burst waveform was used for all neurons. This was generated by measuring the instantaneous spike intervals for the second burst in a consecutive series for all 84 neurons tested (see Fig. 1D) (mean, $90.9 \pm 28.6 \mathrm{~Hz}$ ). We then found a burst with a mean spike frequency and burst duration most similar to that generally recorded and used that as the typical burst waveform. Ionic substitutions and/or ion channel blockers were used to isolate individual currents in response to a command burst waveform presentation. Because of the limitations of the slice preparation and drug delivery times, it was not possible to isolate and characterize multiple ionic currents for each neuron.

In current-clamp experiments, borosilicate pipettes (2-4 M $\Omega$ ) were filled with an internal solution containing the following (in $\mathrm{mM}$ ): 115 K-gluconate, $25 \mathrm{KCl}, 9 \mathrm{NaCl}, 10 \mathrm{HEPES}, 0.2 \mathrm{EGTA}, 1 \mathrm{MgCl}_{2}, 3 \mathrm{~K}_{2}$-ATP, and $1 \mathrm{Na}-\mathrm{GTP}, \mathrm{pH} 7.25$ with $\mathrm{KOH}$, osmolarity adjusted to $280-290$ mOsm. The control external solution consisted of ACSF of the following composition (in mM): $124 \mathrm{NaCl}, 3 \mathrm{KCl}, 1.25 \mathrm{NaH}_{2} \mathrm{PO}_{4}, 26 \mathrm{NaHCO}_{3}, 10$ glucose, $2 \mathrm{CaCl}_{2}$, and $2 \mathrm{MgCl}_{2}$.

In voltage-clamp experiments that focused on isolating sodium currents, $\mathrm{K}^{+}$currents were blocked using an intrapipette solution containing the following (in mM): $130 \mathrm{CsF}, 9 \mathrm{NaCl}, 10$ HEPES, 10 EGTA, 1 $\mathrm{MgCl}_{2}, 3 \mathrm{~K}_{2}$-ATP, and $1 \mathrm{Na}-\mathrm{GTP}$. The external solution contained the following (in mM): $131 \mathrm{NaCl}, 10$ HEPES, $3 \mathrm{KCl}, 10$ glucose, $2 \mathrm{CaCl}_{2}, 2$ $\mathrm{MgCl}_{2}, 10$ tetraethylammonium (TEA)-Cl, $10 \mathrm{CsCl}, 1$ 4-aminopyridine (4-AP), and $0.3 \mathrm{CdCl}_{2}$. Sodium currents were defined by subtraction of the currents remaining in $0.5 \mu \mathrm{M}$ TTX. The internal solution used to isolate $I_{\mathrm{h}}$ current contained the following (in $\mathrm{mM}$ ): $115 \mathrm{~K}$-gluconate, 25 $\mathrm{KCl}, 9 \mathrm{NaCl}, 10$ HEPES, 0.2 EGTA, $1 \mathrm{MgCl}_{2}, 3 \mathrm{~K}_{2}$-ATP, and $1 \mathrm{Na}-\mathrm{GTP}$. The external solution used to isolate $I_{\mathrm{h}}$ currents contained the following (in mM): $124 \mathrm{NaCl}, 3 \mathrm{KCl}, 26 \mathrm{NaHCO}_{3}, 10$ glucose, $2 \mathrm{CaCl}_{2}$, and $2 \mathrm{MgCl}_{2}$, $0.3 \mathrm{CdCl}_{2}$, TTX $(0.5 \mu \mathrm{M}) . I_{\mathrm{h}}$ currents were defined by subtraction of the currents remaining in $10 \mu \mathrm{M}$ 4-ethylphenylamino-1,2-dimethyl-6methylaminopyrimidinium chloride [ZD 7288 (ZD)]. The external solution used to isolate calcium current contained the following (in $\mathrm{mM}$ ): $50 \mathrm{NaCl}, 10 \mathrm{HEPES}, 3 \mathrm{KCl}$, 10 glucose, $2 \mathrm{CaCl}_{2}, 2 \mathrm{MgCl}_{2}, 91$ TEA-Cl, 10 $\mathrm{CsCl}, 14-\mathrm{AP}$, and TTX $(0.5 \mu \mathrm{M})$. Calcium currents were defined by subtraction of the currents remaining in $0.3 \mathrm{mM} \mathrm{CdCl}_{2}$. All drugs were purchased from Sigma (St. Louis, MO) except for ZD 7288 (Tocris, Cookson, MO). Whole-cell recordings were made at room temperature.

Data acquisition and analysis. Currents and voltages were digitized and controlled by pClamp 9.2 software (Molecular Devices). Data were collected and analyzed with a combination of software [Clampfit (ver. 9.2, Molecular Devices), StatView (SAS Institute, Cary, NC), and Microsoft Excel]. Traces shown in some figures were additionally filtered off-line (2000 Hz low-pass, 8-pole Bessel filter). In Fig. 1D, spike frequencies reported in the text are the average of all spikes in the second burst discharge of a series after a depolarizing stimulus. For Figure 9, crosscorrelation analysis was performed by using the voltage peak as the trigger and averaging $20 \mathrm{~ms}$ before and after the trigger for at least four consecutive oscillations before burst onset and repeated three times. To measure the kinetics of the resurgent current, rise time was measured as the time from the end of the step to $+30 \mathrm{mV}$ to the peak of the resurgent current. The decay time constant was obtained from the single exponential fit from the peak of the resurgent current to baseline (Chebyshev method). Results are reported as mean $\pm \mathrm{SD}$, unless indicated. Comparisons between groups of mean values were performed with a paired Student's $t$ test set at a level of significance of $p \leq 0.05$. 


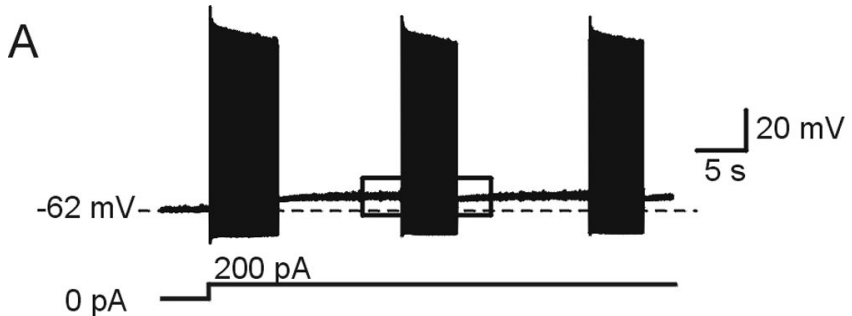

B
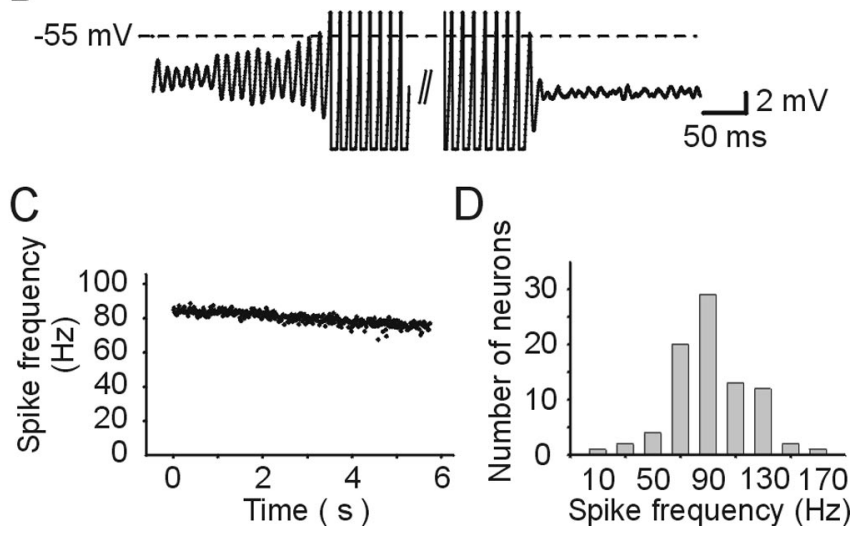

Figure 1. Membrane depolarization induces conditional rhythmical burst discharge in Mes V neurons. $A$, Maintained current injection induces rhythmical burst discharge in Mes $V$ neuron. The top trace shows voltage response, and the bottom trace shows current injection. The dotted line in the voltage trace indicates the resting membrane potential $(-62 \mathrm{mV}) . \boldsymbol{B}$, Traces taken from the boxed region in $\boldsymbol{A}$ on an expanded time and voltage scale. $\boldsymbol{C}$, Instantaneous spike frequency-time relationship for the burst shown in $\boldsymbol{B}$. $\boldsymbol{D}$, Histogram of spike frequency within a burst ( $n=84$ neurons). The mean spike frequency within a burst was obtained from the second burst in a series at threshold stimulus in response to depolarizing current injection.

\section{Results}

Firing properties of mesencephalic trigeminal neurons Electrophysiological recordings were performed on mesencephalic trigeminal neurons from brain slices of rat using the whole-cell patch-clamp recording method. Neurons were recorded throughout the rostral-caudal extent of the nucleus to avoid sampling bias.

In a subset of Mes $\mathrm{V}$ neurons (Wu et al., 2001), rhythmical burst discharge was induced in response to maintained positive current injection in normal internal and external solutions that approximate physiological ionic composition. An example is shown in Figure 1, $A$ and $B$, where depolarization of the membrane from the resting membrane potential produced rhythmical subthreshold oscillations (Fig. $1 B$ ) that grew in amplitude until burst discharge was induced (Fig. $1 A, B$ ). In this example, the mean frequency was $\sim 85 \mathrm{~Hz}$. Typical of these neurons during bursting, there is very little spike adaptation before termination (Fig. 1C) (Wu et al., 2001, 2005). For a subset of neurons, the adaptation ratio (mean spike frequency during the last three ISIs divided by mean frequency during the first ISI was $0.84 \pm 0.07$; $n=84)$. When measured at the threshold of rhythmical burst discharge, the mean intraburst spike frequency for all bursting neurons examined was $90.9 \pm 28.6 \mathrm{~Hz}(14.0-172.7 \mathrm{~Hz} ; n=84)$ (Fig. 1D).

The oscillations and burst discharges do not result from complex interactions between excitatory and inhibitory synaptic inputs because the oscillations and burst discharge were observed in the presence of synaptic channel antagonists, but were blocked after TTX application, suggesting a role for sodium currents as described previously (Wu et al., 2001, 2005)
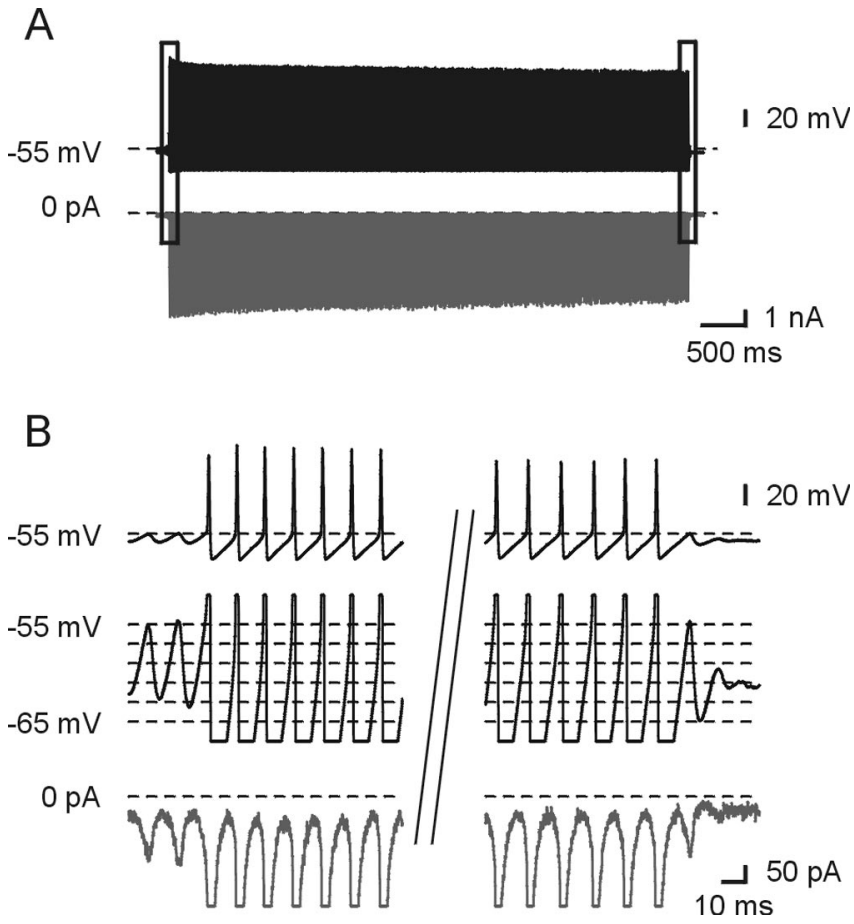

Figure 2. TTX-sensitive sodium currents during burst discharge. $\boldsymbol{A}$, The top trace shows the waveform command recorded in current clamp in a Mes $V$ neuron. The bottom trace shows the subtracted TTX-sensitive current response during voltage clamp. $\boldsymbol{B}$, TTX-sensitive current flowed continuously during a burst. Traces are taken from the boxed segments in $\boldsymbol{A}$ at an expanded time scale. The top and middle traces show the voltage command at an expanded voltage scale. The bottom trace shows the current response. The vertical bars indicate a break in time.

TTX-sensitive sodium currents flow during burst discharge We previously suggested that $I_{\mathrm{NaP}}$ is critical for control of subthreshold and suprathreshold membrane excitability and burst generation in Mes V neuron using pharmacological, electrophysiological, and computer simulation techniques (Wu et al., 2005). However, direct evidence for a role for sodium currents and information regarding the time course of the currents is lacking. Therefore, to obtain direct evidence in support of our hypothesis regarding the role of sodium currents in driving burst discharge, we used the action potential-clamp method to investigate the nature of, and time course for sodium, calcium, and $I_{\mathrm{h}}$ currents during subthreshold membrane oscillations and burst discharge (Raman and Bean, 1999; Do and Bean, 2003; Swensen and Bean, 2003).

To obtain more direct evidence for sodium currents driving burst discharge, we recorded cycles of rhythmical burst discharge from a Mes V neuron in current-clamp mode, and then used the typical burst waveform in voltage-clamp mode as the command protocol (see Materials and Methods). We then recorded TTXsensitive sodium currents obtained by subtraction in response to application of $0.5 \mu \mathrm{M}$ TTX solution (Fig. 2A) (Do and Bean, 2003). As expected, a large sodium current flowed during the upstroke of the action potential and to a lesser degree during the interspike intervals during a burst; this current was also detectable during subthreshold oscillations occurring before and after the burst discharge (Fig. 2B).

Mesencephalic $\mathrm{V}$ neurons exhibit prominent $I_{\mathrm{h}}$ current (Khakh and Henderson, 1998; Tanaka et al., 2003) and both lowand high-threshold calcium currents (our unpublished observations) that could participate in production of net inward current 
A

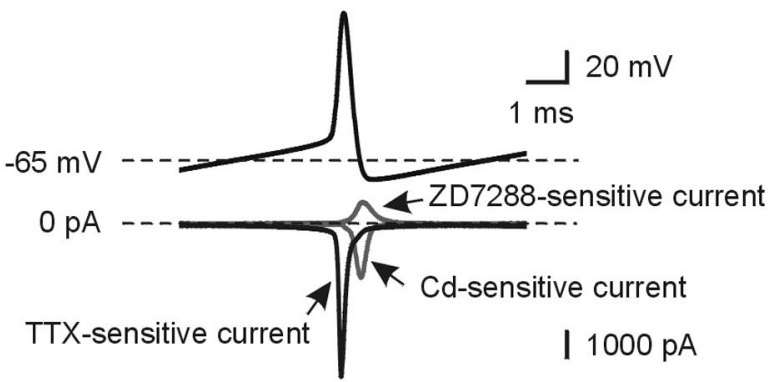

B
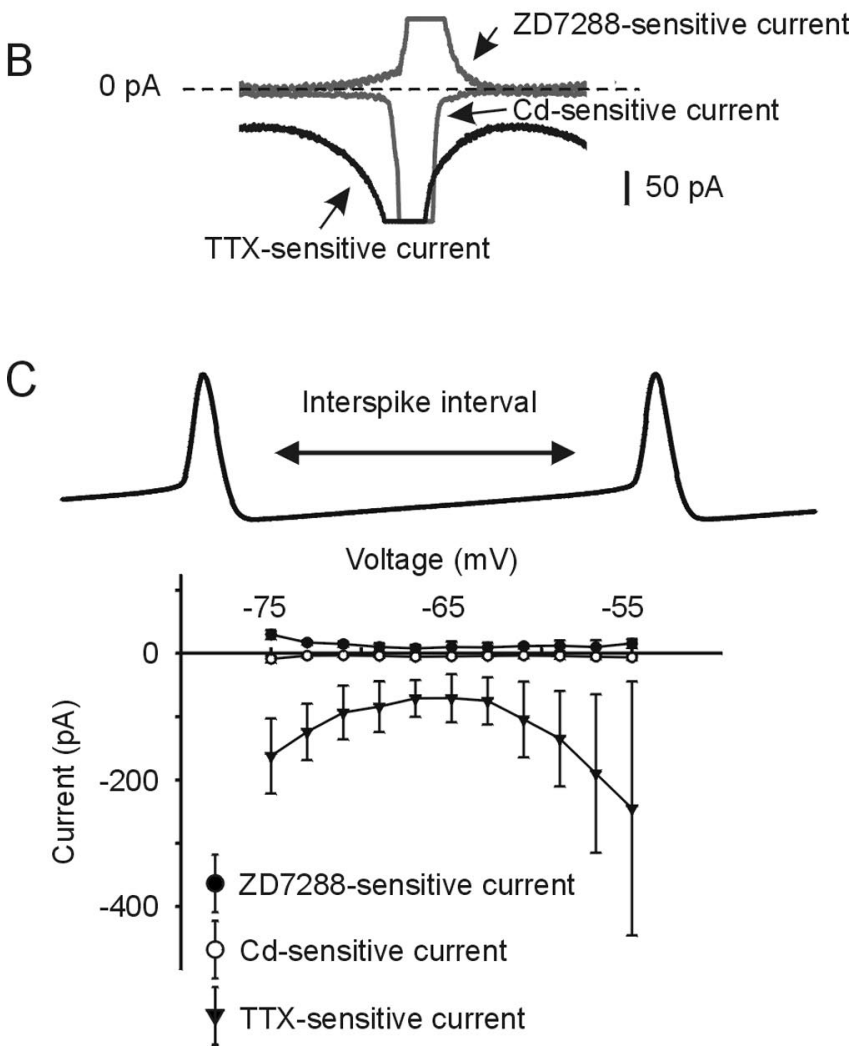

Figure 3. Sodium current, in contrast to $I_{\mathrm{h}}$ and calcium currents, is the predominate current during the interspike interval. $\boldsymbol{A}$, Signal-averaged spike waveform command (top) and current responses (bottom) for sodium, calcium, and $I_{\mathrm{h}}$ currents. Individual spikes for each current were aligned at their peaks, and the spikes and their currents were averaged in response to each spike within a burst. $\boldsymbol{B}$, Traces taken from data in $\boldsymbol{A}$ at an expanded current scale. Note peak amplitudes of voltage and current are off scale. $\boldsymbol{C}$, Current-voltage relationship during the interspike interval for each current is shown. Error bars indicate \pm SD. TTX-sensitive $I_{\mathrm{Na}}$ (triangle, $n=10$ ), ZD 7288-sensitive $I_{\mathrm{h}}$ (solid circle, $n=3$ ), $\mathrm{Cd}^{2+}$ sensitive $I_{\mathrm{Ca}}$ (open circle, $n=6$ ) are shown.

to drive spike activity during burst discharge. Using the action potential-clamp method, we determined the time course and extent of current flow through those channels. $I_{\mathrm{h}}$ was determined by subtraction of the recording in response to application of $10 \mu \mathrm{M}$ external ZD 7288 from control (Pape, 1996; Beurrier et al., 2000; Tanaka et al., 2003). Figure $3 A$ shows the action potential command waveform obtained from the average of consecutive action potentials in a burst and the resulting subtracted ZD-sensitive current, whereas Figure $3 B$ shows the same data at higher gain. In all three neurons tested, the ZD-sensitive current was very small and outward during the interspike interval (between -75 and $-55 \mathrm{mV}$ ) and largest during the repolarization phase of the action potential. The mean values for this current flowing during the interspike interval were $30.0 \pm 7.2 \mathrm{pA}$ at $-75 \mathrm{mV}, 10.3 \pm 5.9$ $\mathrm{pA}$ at $-69 \mathrm{mV}, 9.6 \pm 7.5 \mathrm{pA}$ at $-63 \mathrm{mV}$, and $15.9 \pm 7.1 \mathrm{pA}$ at $-55 \mathrm{mV}(n=3)$. These results suggest that only $\sim 10 \mathrm{pA} \mathrm{ZD-}$ sensitive current flows during the interspike interval, which is not $I_{\mathrm{h}}$. Figure 3, $A$ and $B$, shows that, during the falling phase of the spike, there is an outward ZD-sensitive current, which probably reflects a small, nonspecific block of undetermined $\mathrm{K}^{+}$channels (Do and Bean, 2003), because we previously observed a small reduction in afterhyperpolarization (AHP) after drug application (Tanaka et al., 2003).

Calcium currents flowing during a burst were determined by subtraction using by $0.3 \mathrm{mM} \mathrm{CdCl}_{2}$ solution, which contained 91 mM TEA and $0.5 \mu \mathrm{M}$ TTX. Figure $3, A$ and $B$, shows the $\mathrm{Cd}^{2+}$ sensitive inward currents. Although prominent inward calcium current was evident during the spike, it was very small during the interspike interval. The average calcium current flowing during the interspike interval was $-8.6 \pm 5.4 \mathrm{pA}$ at $-75 \mathrm{mV},-3.8 \pm 1.6$ $\mathrm{pA}$ at $-69 \mathrm{mV},-3.9 \pm 2.4 \mathrm{pA}$ at $-63 \mathrm{mV}$, and $-6.2 \pm 4.0 \mathrm{pA}$ at $-55 \mathrm{mV}(n=6)$. A summary of the amplitudes and time courses of the sodium, calcium, and ZD-sensitive currents flowing during the interspike interval is shown in Figure $3 C$. Individual spikes within the entire burst discharge were aligned at their peaks, and the spikes and their currents were averaged over multiple current cycles. Clearly, from the data, we conclude that the most prominent inward current during the ISI is carried by sodium, with minor contribution from calcium currents and no contribution from $I_{\mathrm{h}}$.

\section{Properties of TTX-sensitive sodium current}

In addition to $I_{\mathrm{NaT}}, I_{\mathrm{NaP}}$ is present in many neuron types, including Mes V neurons (Wu et al., 2001, 2005), and more recently, a resurgent sodium current component has been characterized in Purkinje neurons (Raman and Bean, 1997). It is believed that this current results during action potential repolarization from recovery of inactivated channels through "open states." To determine whether these currents contribute to the inward current during the interspike interval, we performed the following experiments using modified voltage-clamp protocols described previously (Do and Bean, 2003).

Sodium currents were obtained using the standard action potential-clamp waveform during a burst in real time, and during a modified condition in which the same action potential burst waveform was slowed down by a factor of 100 to inactivate the fast $I_{\mathrm{NaT}}$. In this modified protocol, the rising rate of the membrane voltage is slow enough to ensure that complete fast inactivation of $I_{\mathrm{NaT}}$ is obtained at each voltage cycle, leaving only TTXsensitive $I_{\mathrm{NaP}}$. Figure $4 \mathrm{~A}$ shows at low gain the action potential waveform and underlying sodium currents, whereas Figure $4 B$ shows two spikes within a burst in $100 \times$ slow time and the underlying sodium current. In this case, during the spikes, the large fast inward $I_{\mathrm{NaT}}$ is, indeed, abolished. For comparison, preceding the two slowed action potentials is the real time $(1 \times$ time $)$ action potential waveform for two spikes and the large underlying $I_{\mathrm{NaT}}$ component below. Figure $4 \mathrm{C}$ shows the same two action potential templates and the associated $100 \times$ slow-time currents compressed to real time at higher gain. Superimposed are the realtime currents for comparison. As is evident, during the repolarization phase, the sodium current is very small and is not flowing at all during the early part of the AHP when evoked in $100 \times$ time. However, starting around the first one-third of the interspike interval and continuing until the next spike, a slowly developing $I_{\mathrm{NaP}}$ flowed and increased in amplitude. The real-time sodium currents are large during the action potential and, as expected, flow continuously during the interspike interval (Fig. $4 C)$. A plot of conductance of this slow current obtained from the $I-V$ relationship during the interspike interval is shown in Figure 
A
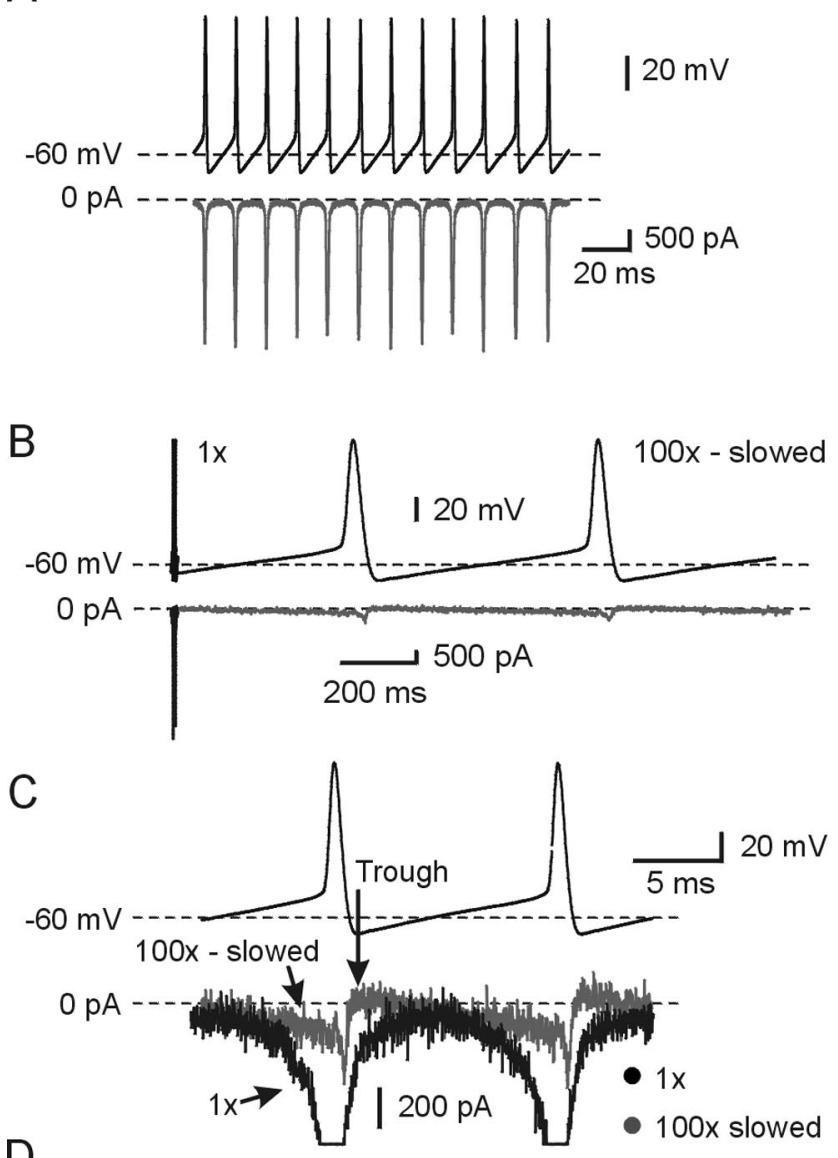

$\mathrm{D}$

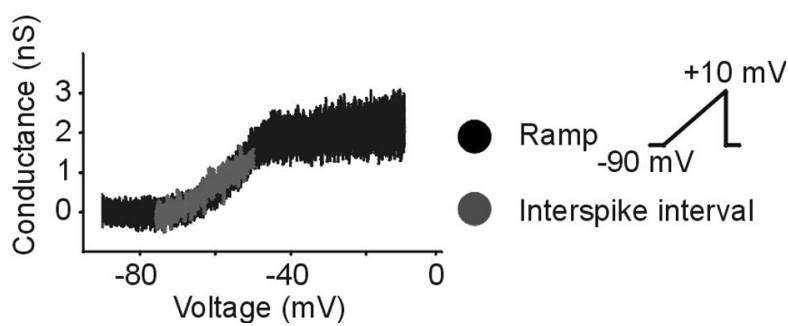

Figure 4. Isolation of persistent sodium current from total sodium current. $A$, Voltage waveform (top) and TTX-sensitive sodium current response (bottom). $B$, TTX-sensitive $I_{\text {NaP }}$ was isolated by the artificial waveform protocol (see Materials and Methods). The first two spikes were elicited in natural time, followed by two spikes elicited at $100 \times$ slower than natural time (slowed time). This effectively inactivated $I_{\mathrm{NaT}}$ and exposed $I_{\mathrm{NaP}}$. C, TTX-sensitive $I_{\mathrm{Nap}}$ recorded during the template waveform command in $\boldsymbol{B}$ was plotted in 100 -fold compressed time base and superimposed with TTX-sensitive total sodium current obtained in natural time. Note that these two traces overlapped around the middle of the interspike interval. $\boldsymbol{D}$, Comparison of conductance-voltage relationship of $/_{\text {NaP }}$ obtained by the slowed spike protocol method above and the voltage ramp protocol ( $33.3 \mathrm{mV} / \mathrm{s}$; gray) are shown. Conductance-voltage relationships were plotted as a function of command potential (see Materials and Methods) $\left(E_{\text {rev }}\right.$ was calculated to be $+45 \mathrm{mV}$ ).

$4 D$ (gray). It is likely that this slow current observed during the interspike interval is the traditional "persistent" slowly inactivating sodium current, because its conductance overlaps with the persistent current evoked by a traditional slow ramp voltageclamp command $(33.3 \mathrm{mV} / \mathrm{s})$ over a similar voltage range (Fig. $4 D)$. These results were observed in five of five neurons examined.

During the latter part of spike repolarization and the early part of the AHP, a large sodium current flows when evoked in real time (Fig. 4C, black). However, during the early part of the AHP,
A1
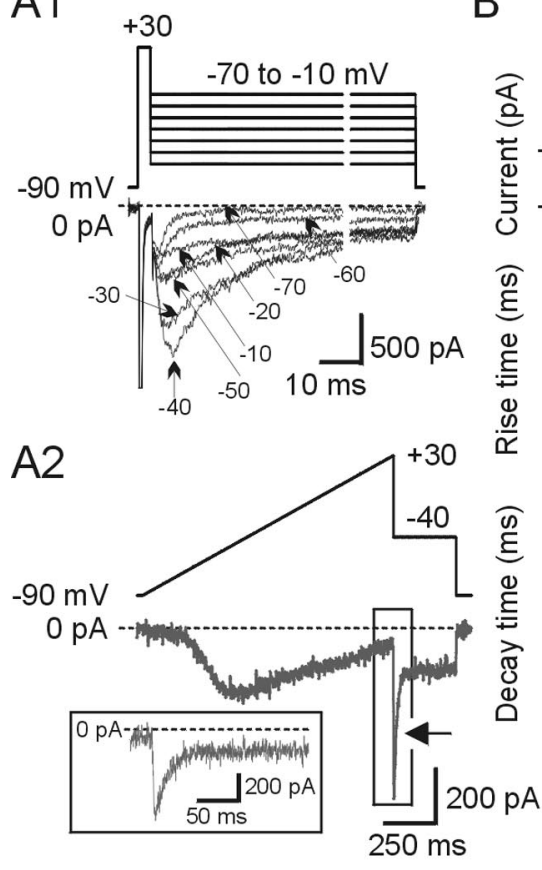

B
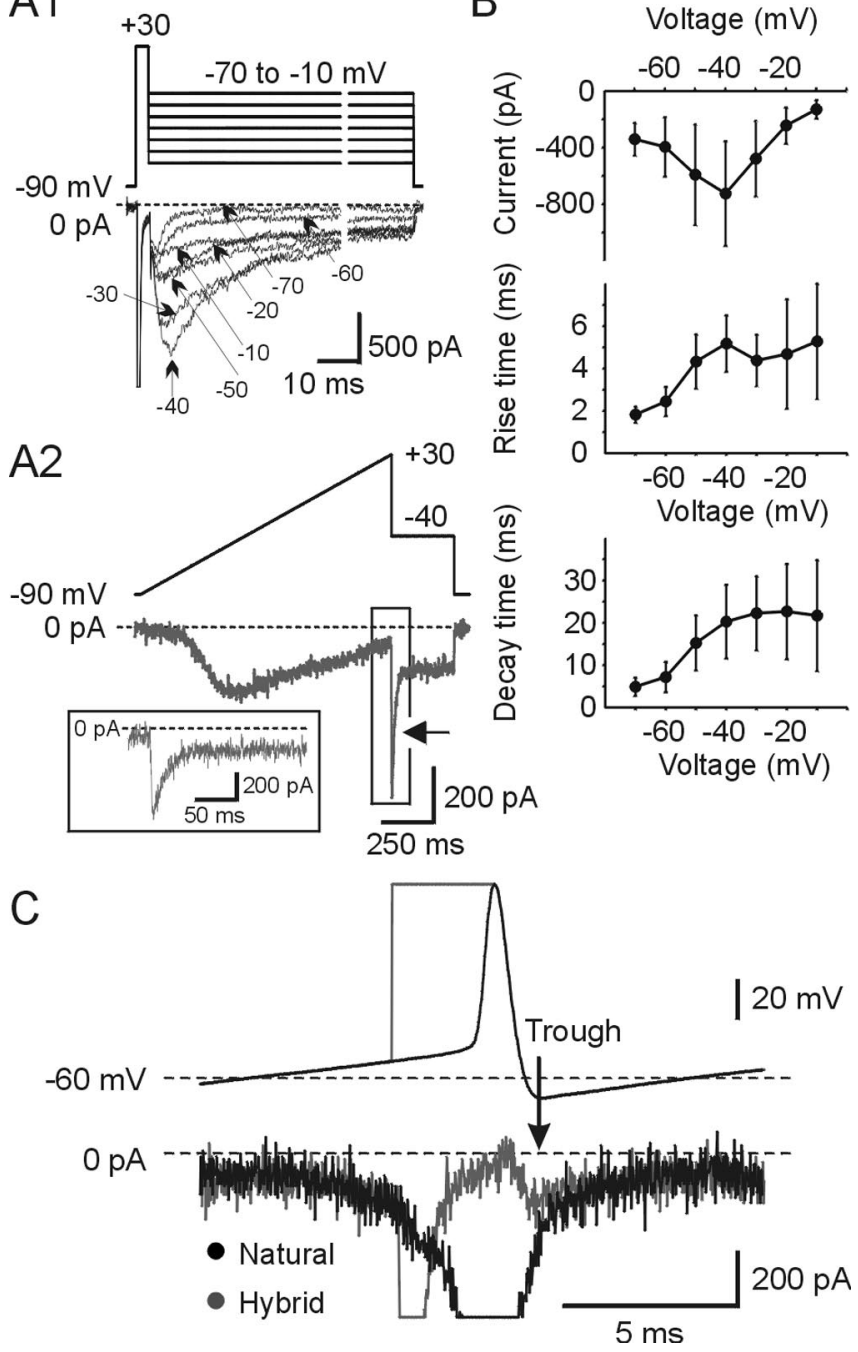

Figure 5. Mes $V$ neurons show resurgent sodium currents. $\boldsymbol{A} 1$, The top trace shows voltage protocol, and the bottom trace shows current response. $I_{\text {NaT }}$ was evoked by a 3 ms step pulse from -90 to $+30 \mathrm{mV}$. TTX-sensitive $I_{\text {NaR }}$ was elicited when the membrane was repolarized to voltages between -70 and $-10 \mathrm{mV}$ after maximal fast inactivation. The data have been expanded for clarity, and the break in time is $10 \mathrm{~ms}$. $\mathbf{A 2}$, TTX-sensitive sodium current was obtained by applying a slow ramp ( -90 to $+30 \mathrm{mV} ; 100 \mathrm{mV} / \mathrm{s}$ ) followed by a step repolarization to $-40 \mathrm{mV}$. $I_{\text {NaR }}$ is evident after repolarization to $-40 \mathrm{mV}$ (arrow). The inset shows the expanded timescale of the boxed region in which $I_{\text {NaR }}$ is present. $B, T T X$-sensitive $I_{\text {NaR }}$ properties are voltage dependent. Relationships between peak $I_{\text {NaR }}$ (top), rise time (middle), and decay time (bottom) versus repolarization command potential are shown. Error bars indicate SD. C, Relationship between resurgent sodium current components and total sodium current. The hybrid waveform protocols are shown on the top, and the current traces are shown below. Solid black is total sodium current evoked by template action potential, and the gray trace is current in response to a step pulse $(3 \mathrm{~ms})$ to inactivate $I_{\mathrm{NaT}}$ followed by the repolarization phase of the action potential. The isolated $I_{\mathrm{NaR}}$ (gray) was superimposed with the total sodium current (black). Note that these two traces overlapped at the peak of the AHP and for some time thereafter.

as shown, $I_{\mathrm{NaP}}$ is not flowing. Therefore, the sodium current flowing during this period in real time must be attributable to a contribution from an additional sodium component, such as a resurgent sodium current or a sodium tail current (Do and Bean, 2003) or both. To distinguish between these components, modified action potential waveforms were used.

Figure $5 A 1$ shows the protocol used to determine the presence of $I_{\mathrm{NaR}}$ and underlying sodium currents obtained by subtraction for a family of command waveforms. After a step command to 
$+30 \mathrm{mV}$ for $3 \mathrm{~ms}$, sufficient to produce complete fast inactivation of $I_{\mathrm{NaT}}$, subsequent waveforms to voltages between -70 and -10 $\mathrm{mV}$ were applied. As shown, after repolarization to the new command potential, an inward current with maximal amplitude around $-40 \mathrm{mV}$ is seen. The peak current amplitude of $I_{\mathrm{NaR}}$ was similar even when the period of prepulse was changed from 3 to $10 \mathrm{~ms}(n=4)$ (data not shown). The peak $I_{\mathrm{NaR}}$ at $-40 \mathrm{mV}$ was $-725.8 \pm 370.4 \mathrm{pA} / \mathrm{pF}$ and occurred in $5.1 \pm 1.3 \mathrm{~ms}$, and decayed with a time constant tau of $20.2 \pm 8.7 \mathrm{~ms}(n=23)$. Figure $5 \mathrm{~B}$, middle and bottom, show both the rise and decay times as a function of voltage. The properties of this "resurgent current" are quite similar to those reported previously for Purkinje neurons (Raman and Bean, 1997; Do and Bean, 2003). At voltages more negative than $-40 \mathrm{mV}$, the peak $I_{\mathrm{NaR}}$ became smaller as shown in the plot of peak current versus potential (Fig. $5 B$, top). This is not consistent with a sodium tail current, because the $I-V$ relationship is not ohmic with positive slope as expected for a tail current. It is also unlikely that this represents a persistent sodium tail current, because the current flowing immediately after an instantaneous repolarization to $-40 \mathrm{mV}$ from a preceding ramp command to $+30 \mathrm{mV}$, typically used to elicit $I_{\mathrm{NaP}}$ (Wu et al., 2001, 2005), is larger than the peak current observed during the ramp at $-40 \mathrm{mV}$ (Fig. 5A2).

To determine the contribution of $I_{\mathrm{NaR}}$ to the total sodium current during spike repolarization and the early part of the AHP, we removed the contribution of the sodium tail current that flows at that time using modified protocols (Do and Bean, 2003). Figure $5 C$ shows two voltage command waveform protocols (top traces) superimposed and the resulting sodium currents (bottom traces). Using the action potential waveform in real time, the total inward sodium current is very large (truncated in figure). At the peak of the AHP, this current is $\sim 150 \mathrm{pA}$ (Figs. $3 C, 5 C$ ). Superimposed is a command waveform designed to produce maximal inactivation of the fast $I_{\mathrm{NaT}}$ and hence no tail current. After the depolarization to $+33 \mathrm{mV}$ for $3 \mathrm{~ms}$, the same repolarization spike waveform used previously was then applied. As shown, during these conditions, only a small inward sodium current remains during the peak of the AHP and shortly thereafter. Furthermore, the current flowing at the peak and after the AHP for both conditions overlapped. This current must be resurgent sodium current, because during this time the tail current is eliminated, and from the preceding experiment (Fig. $4 C$ ), $I_{\mathrm{NaP}}$ flowing during this time is zero. This was observed in all five cells examined.

\section{Slow inactivation of TTX-sensitive sodium currents}

Previously, we suggested that burst termination could result from accumulation of slow inactivation of sodium currents during a burst (Wu et al., 2001, 2005). To obtain evidence for this hypothesis, we performed experiments using the action potential-clamp to determine whether $I_{\mathrm{NaT}}, I_{\mathrm{NaR}}$, and $I_{\mathrm{NaP}}$ are susceptible to slow inactivation using protocols designed to isolate these currents.

Figure $6 \mathrm{~A}$ shows the command burst waveform (top) obtained in current clamp, and the underlying current flow during a burst (bottom trace) during voltage clamp. Figure $6 B$ shows an expanded view obtained from Figure $6 A$. $I_{\mathrm{NaP}}$ was defined as the average current flowing over a $1 \mathrm{~ms}$ time window $5 \mathrm{~ms}$ after the AHP trough (Fig. 6B, box), when resurgent and tail currents are minimal. The mean of $I_{\mathrm{NaP}}$ was calculated for the first and last five spikes within a burst. We then compared the average of $I_{\mathrm{NaP}}$ at this early time with that measured just before burst termination. Based on 10 neurons examined, the mean amplitude of the $I_{\mathrm{NaP}}$ at the end of the discharge was reduced to $\sim 86.1 \pm 11.3 \%$ of control compared with the mean amplitude at the beginning $(p<0.03$;
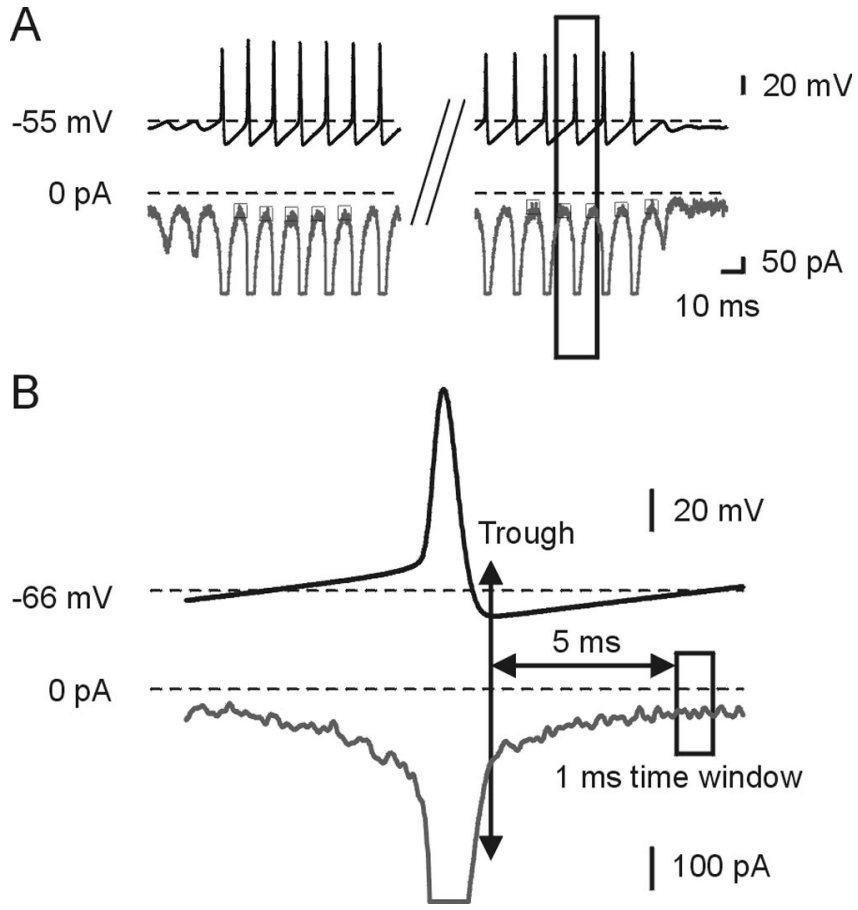

Figure 6. Slow inactivation of $I_{\mathrm{NaP}}$ during a burst. $A$, The top trace shows the early part (left side) and late (right side) of the burst template voltage command at an expanded timescale. The bottom trace shows the current responses. The mean currents were calculated for the first five spikes and the last five spikes during the burst discharge. The small boxes during each ISI indicate the region in which currents were measured. $\boldsymbol{B}$, The traces show the boxed segments in $\boldsymbol{A}$ at an expanded timescale. The current amplitude was calculated by averaging the current flowing over a $1 \mathrm{~ms}$ window $5 \mathrm{~ms}$ after the trough.

$n=10$ ), suggesting that a reduction in $I_{\mathrm{NaP}}$ during a maintained burst would contribute, modestly, to burst termination (Wu et al., 2005).

However, additional mechanisms, such as slow inactivation of the $I_{\mathrm{NaT}}$ and $I_{\mathrm{NaR}}$ (Fleidervish and Gutnick, 1996; Do and Bean, 2003) could contribute to burst termination, as well. To test this hypothesis, we used a modified protocol that allowed us to measure all three currents simultaneously (Fig. 7A) (Do and Bean, 2003) in solutions containing a reduced concentration of external sodium $(50 \mathrm{~mm} \mathrm{NaCl}$ and $91 \mathrm{~mm} \mathrm{TEA}-\mathrm{Cl})$ to minimize series resistance errors. Figure $7 A$ shows the protocol and the underlying sodium currents. Voltage-dependent slow inactivation of $I_{\mathrm{NaT}}, I_{\mathrm{NaR}}$, and $I_{\mathrm{NaP}}($ Fig. $7 A$ ) were tested using prepulses of $20 \mathrm{~s}$ duration from -90 to $-40 \mathrm{mV}$ followed by a return to $-90 \mathrm{mV}$ for $100 \mathrm{~ms}$ to allow recovery from fast inactivation. The different sodium current components were evoked by using a $10 \mathrm{~ms},+15$ $\mathrm{mV}$ step followed by a return to $-40 \mathrm{mV}$ for $100 \mathrm{~ms}$. $I_{\mathrm{NaT}}$ was defined as peak minus steady-state current during the step to +15 $\mathrm{mV} . I_{\mathrm{NaR}}$ was defined as peak minus steady-state current (taken at end of pulse) evoked during the step to $-40 \mathrm{mV}$, whereas $I_{\mathrm{NaP}}$ was defined as steady-state current at the end of the pulse minus baseline current during the step to $-40 \mathrm{mV}$. Figure $7, B$ and $C$, shows a summary of steady-state inactivation for each of the currents by plotting relative steady-state conductance versus prepulse potential in low external sodium $(50 \mathrm{~mm})$ and normal sodium (131 mM) conditions. Regardless of external sodium concentration, $I_{\mathrm{NaP}}$ was most susceptible to slow inactivation followed by $I_{\mathrm{NaR}}$, then $I_{\mathrm{NaT}}$ (measured only in low sodium) (Fig. $7 \mathrm{~B}, \mathrm{C})$. For comparison, fast inactivation of the $I_{\mathrm{NaT}}$ obtained in $15 \mathrm{~mm}$ external sodium is also shown (Fig. $7 B$, dotted line). The 
mean relative TTX-sensitive peak conductance was plotted against the conditioning pulse potential and fitted with a Boltzmann equation. In low sodium, the fit parameters were $V_{1 / 2}=-65.0 \pm 6.3 \mathrm{mV}$ and $k=11.9 \pm 2.0 \mathrm{mV}$ for $I_{\mathrm{NaT}}, V_{1 / 2}=$ $-73.3 \pm 6.4 \mathrm{mV}$ and $k=14.4 \pm 6.3 \mathrm{mV}$ for $I_{\mathrm{NaR}}(n=6)$, and $V_{1 / 2}=-72.7 \pm 9.1$ $\mathrm{mV}$ and $k=14.1 \pm 6.1 \mathrm{mV}$ for $I_{\mathrm{NaP}}$. In normal sodium, the Boltzmann fit parameters yielded $V_{1 / 2}=-63.6 \pm 6.9 \mathrm{mV}$ and $k=8.4 \pm 2.6 \mathrm{mV}$ for $I_{\mathrm{NaR}}, V_{1 / 2}=-65.0 \pm$ $3.0 \mathrm{mV}$ and $k=7.7 \pm 1.4 \mathrm{mV}$ for $I_{\mathrm{NaP}}(n=$ $8)$. In low sodium and measured at -40 $\mathrm{mV}$, each relative conductance was reduced to $48.7 \pm 16.8 \%\left(I_{\mathrm{NaT}}\right), 43.9 \pm 5.6 \%$ $\left(I_{\mathrm{NaR}}\right)$, and $28.2 \pm 17.9 \%\left(I_{\mathrm{NaP}}\right)$, respectively, whereas in normal sodium $I_{\mathrm{NaR}}$ relative conductance was reduced to $59.9 \pm$ $10.7 \%$ and $I_{\mathrm{NaP}}$ relative conductance was reduced to $43.0 \pm 17.7 \%$.

To confirm that the voltage was adequately space clamped during fast $I_{\mathrm{NaT}}$ evoked by the hybrid protocol used in Figure 7 , we performed additional experiments using the outside-out patch configuration in $50 \mathrm{~mm}$ external sodium solution. With this configuration, space clamp errors are eliminated. We compared the $I-V$ relationship of the fast $I_{\mathrm{NaT}} \mathrm{ob}-$ tained in whole-cell configuration with that obtained in the same cell after entering the outside-out configuration. The $\mathrm{P} / \mathrm{N} 4$ method was used for leak subtraction. Figure $7 D$ shows the summary $I-V$ relationship superimposed for the two conditions on their respective current scales. The adequacy of the whole-cell configuration to clamp fast $I_{\mathrm{NaT}}$ in low external sodium is confirmed by reasonably good, but not perfect, overlap of the $I-V$ relationship for both recording methods and, in particular, the similar reversal potentials. The fact that Mes V neurons are oval and devoid of dendritic processes further enhance our confidence in use of the hybrid protocol in the whole-cell configuration to clamp the fast currents in low sodium recording conditions and most likely account for the relatively small differences in the $I-V$ relationships between the two conditions.

To obtain additional evidence that slow inactivation of these currents contributes to burst frequency and termination, we applied the test protocol described in Figures $7 A$ and $8 A$ at various times after the onset of a burst waveform command (Fig. $8 \mathrm{~A}$ ) initially obtained in current clamp. In this situation, the spike activity during the burst command that preceded the test protocol effectively served as the conditioning prepulse. These data were obtained in low sodium to allow voltage control of the action potential command template. Control data for the time dependence of onset of slow inactivation were first analyzed by means of a step protocol before running the burst waveform command (Fig. $8 \mathrm{~A}$, inset). After this, the duration of burst discharge was artificially changed and the test protocol was induced after each modified burst (Fig. $8 \mathrm{~A}$, right; numbers indicated onset of test protocol). We then compared the amplitude change of these currents before, and after the burst waveform. To avoid trial-to-trial accumulation of slow inactivation, the protocol was
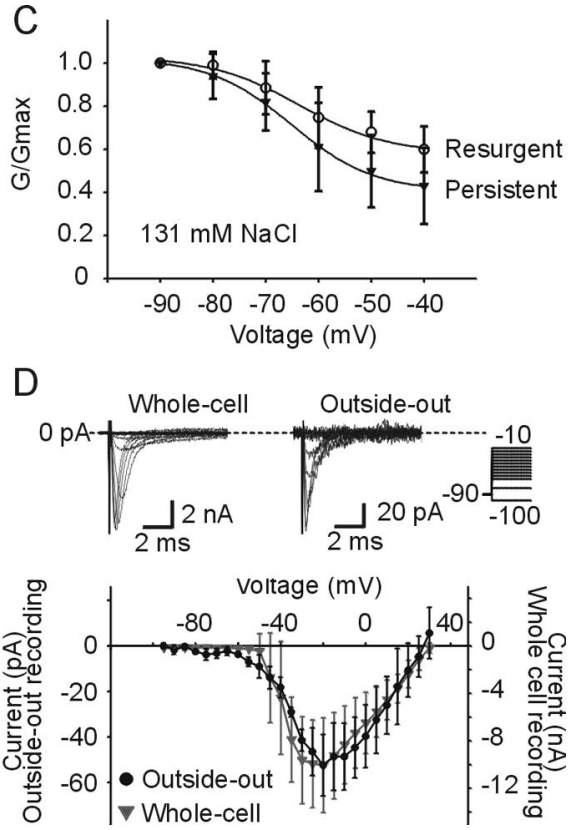

Figure 7. Sodium current components show voltage-dependent slow inactivation. $\boldsymbol{A}$, Hybrid protocol to demonstrate slow Representative current traces obtained from voltage steps between -95 and $+35 \mathrm{mV}$ from a holding potential of $-90 \mathrm{mV}$ are currents for both recording modes (bottom). All currents were leak subtracted (see Materials and Methods). The gray line shows outside-out recording, and the black line shows whole-cell recording $(n=3)$. Error bars indicate SD.

repeated every $10 \mathrm{~s}$. A test step protocol applied $100 \mathrm{~ms}$ after the termination of the waveform command (to remove fast inactivation) revealed that all of the three sodium currents were depressed compared with control. Figure $8 B$ indicates that some degree of slow inactivation develops as the burst duration is prolonged for all 3 currents. Furthermore, the development of slow inactivation accompanied the slow spike adaptation as indicated by the superimposed relative instantaneous spike frequency of the burst template used (Fig. $8 \mathrm{~B}$ ). Similar to that shown in Figure 7, $I_{\mathrm{NaP}}$ was most susceptible to slow inactivation. Thus, the present data are compatible with the hypothesis that burst termination and slow spike adaptation results, to some degree, from slow inactivation of $I_{\mathrm{NaT}}, I_{\mathrm{NaR}}$, and $I_{\mathrm{NaP}}$ sodium currents.

\section{Persistent sodium current flows during the interburst interval}

Typically, bursts terminate abruptly with a depolarizing afterpotential and damped oscillations that do not trigger a spike. After burst termination, subthreshold oscillations are smallest compared with those occurring just before burst onset. Interestingly, the steady-state membrane potential at burst termination is only a few millivolts hyperpolarized compared with burst onset (Figs. $1 B, 9 B$ ) (Wu et al., 2001). During the interburst period, the membrane potential slowly depolarizes and subthreshold oscillations are reinitiated and give rise to burst onset. To determine whether slow sodium currents develop during this time period, we measured the TTX-sensitive current that flows during the interburst 


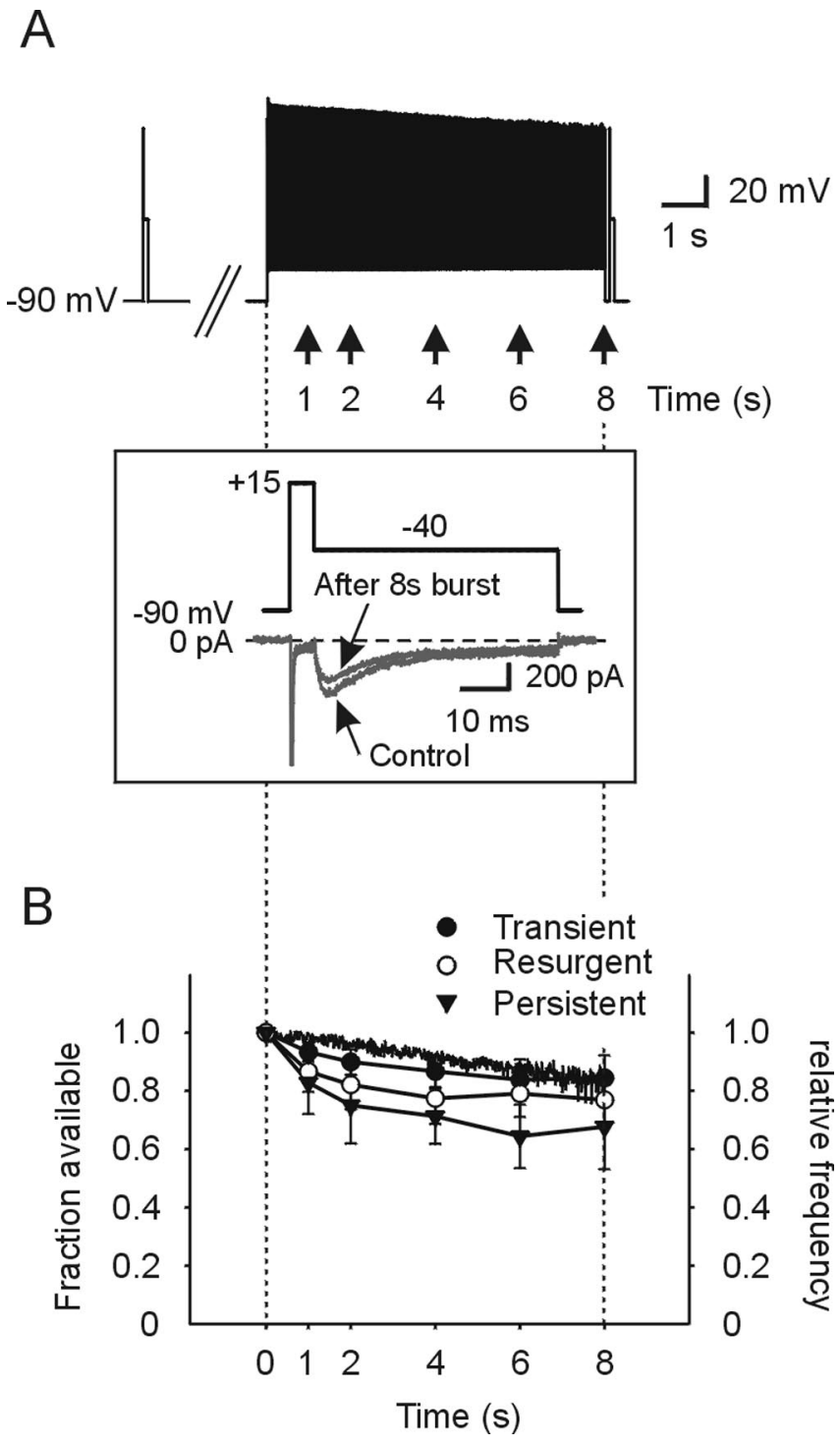

Figure 8. Time course of slow inactivation for sodium currents during a burst. $A$, Protocol to examine time course of slow inactivation for sodium current components during burst discharge. Hybrid test protocol shown (inset, top; voltage, bottom; current) was applied at the time points indicated during a command template burst waveform. The hybrid command template was the same as shown in Figure $7 \mathrm{~A}$ except that the prepulse was now the preceding spike discharge. Same step command protocol was tested as a control $10 \mathrm{~s}$ before the burst waveform. $B$, Time course of slow inactivation of $I_{\mathrm{NaT}}, I_{\mathrm{NaR},}$ and $I_{\mathrm{NaP}}$ during the burst discharge. Relative currents are plotted versus time after the burst template was applied for each current component. Relative spike frequency during the template burst is plotted on the same timescale for comparison (continuous trace). Error bars indicate SD.

period. Figure $9 A$ shows the burst template used as the voltage command and the subsequently recorded TTX-subtracted sodium current at low gain, whereas Figure $9 B$ shows the same data obtained within the area depicted in Figure $9 A$ (box) at higher gain. As is evident, during the interburst interval, as the template voltage command depolarized the membrane potential a slow sodium current developed. Close inspection of the current traces during this time show the presence of small current oscillations that get progressively larger with time (Fig. 9C). To determine the relationship between the voltage and current oscillations, crosscorrelation analysis was performed. The peak of the voltage oscillation was used as the reference trigger and the subsequent current was averaged during an approximately $\pm 20 \mathrm{~ms}$ window
A

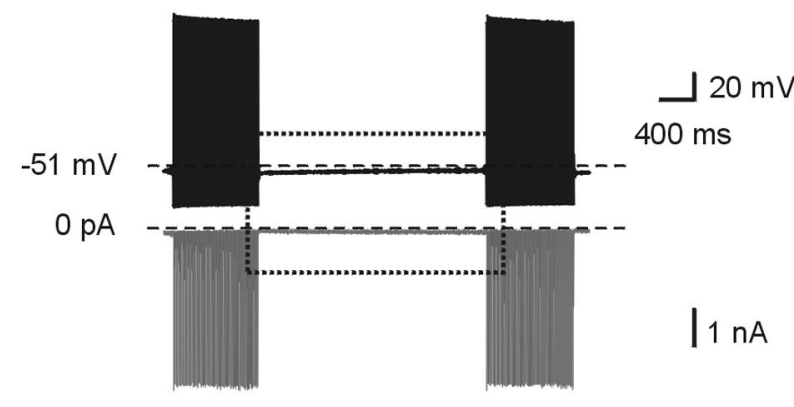

B

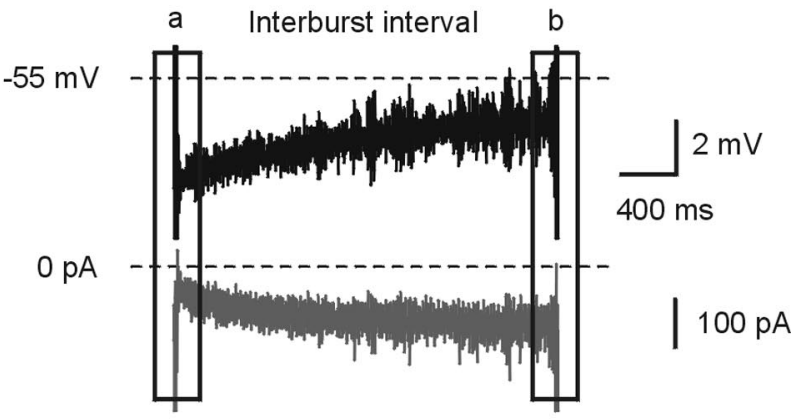

C

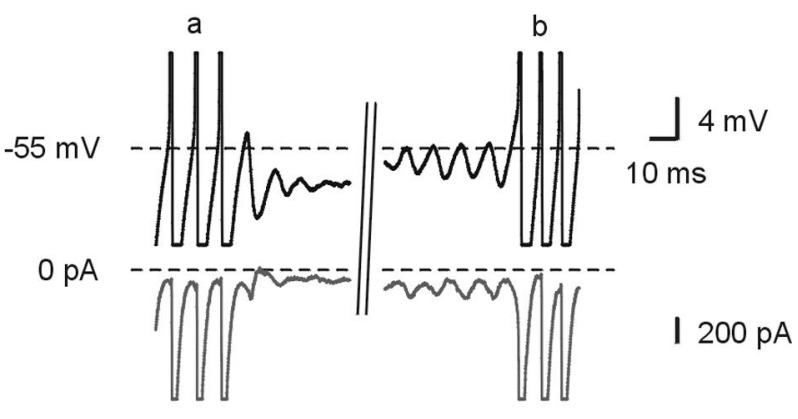

D

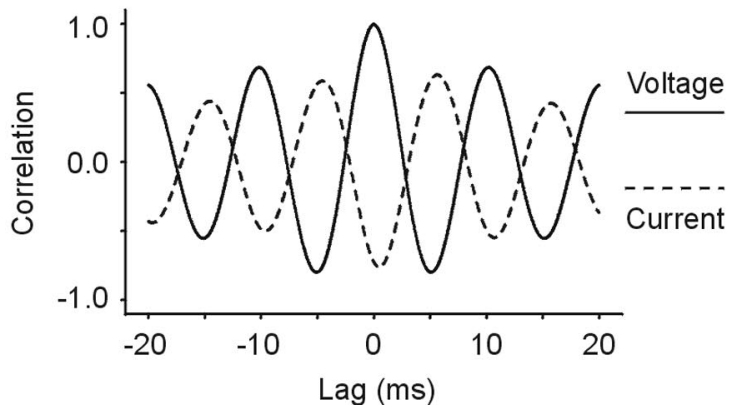

Figure 9. Time course of development of persistent sodium current during the interburst interval. $A$, The top trace shows the waveform command. The dotted line indicates $-51 \mathrm{mV}$. The bottom trace shows a subtracted TTX-sensitive current response at low gain. $\boldsymbol{B}$, The traces show the dotted boxed segment in $A$ at high gain. The top trace shows the voltage command, and the bottom trace shows the subtracted TTX-sensitive current. During the interburst voltage trajectory, the amplitude of TTX-sensitive sodium current increased exponentially. C, Onset and termination of burst discharge at high gain. The traces show the boxed segments in $\boldsymbol{B}$ at an expanded timescale. $\boldsymbol{D}$, Cross-correlation analysis between subthreshold voltage oscillations (reference trigger) and current responses. Downward deflection of current indicates inward current.

around each oscillation before burst discharge. Figure $9 D$ shows that, in fact for each subthreshold membrane potential oscillation, a fast activating and deactivating inward sodium current is generated and associated the voltage command. 


\section{Discussion}

The present study is the first detailed examination of the contribution of various components of sodium current to Mes V burst discharge. Specifically, we demonstrate that components of the total sodium current $\left(I_{\mathrm{NaT}}, I_{\mathrm{NaR}}, I_{\mathrm{NaP}}\right)$ contribute at specific time points to the membrane potential trajectory during both the intraburst interspike interval and interburst membrane potential periods and are the major inward currents supporting burst discharge. Furthermore, the data indicate that slow inactivation of not only $I_{\mathrm{NaP}}$, as suggested previously (Wu et al., 2005), but $I_{\mathrm{NaT}}$ and $I_{\mathrm{NaR}}$ contribute to spike frequency adaptation and burst termination.

\section{Contribution of sodium current components to spike discharge}

Previous studies have successfully applied the action potentialclamp method to analyze the underlying ionic currents responsible for pacemaking and burst generation in different types of neurons (Llinas et al., 1982; Raman and Bean, 1997; Do and Bean, 2003; Swensen and Bean, 2003; among others). Our previous studies indirectly characterized the ionic currents responsible for trigeminal sensory and motoneuronal excitability based on pharmacological dissection (Hsiao et al., 1998; Wu et al., 2001, 2005; Tanaka et al., 2003). Although useful, as pointed out previously (Swensen and Bean, 2003), with that type of analysis, the interpretation can be misleading, because blocking one conductance can alter the subsequent voltage trajectory and underlying conductance of another ion channel. The action potential-clamp method uses a template of the actual voltage trajectory obtained in current clamp and allows for direct measurement of the underlying currents. Therefore, this method allowed us to precisely determine the contribution and time course of the different sodium current components during burst generation more accurately. Fortunately, we were able to apply this method to the slice preparation because these neurons lack dendrites, and are oval in shape, and are less prone to voltage-clamp errors (Fig. 7).

Sodium, as opposed to calcium or $I_{\mathrm{h}}$ currents, is the main charge carrier flowing just before, and during, burst discharge. Other studies have demonstrated the importance of either $I_{\mathrm{h}}$ currents or calcium currents in driving repetitive discharge or bursting (Pape, 1996; Jackson et al., 2004). We showed that block of $I_{\mathrm{h}}$ currents in Mes $\mathrm{V}$ neurons reduces the duration of a spike train evoked by a current pulse when initiated from hyperpolarized membrane potentials ( $-65 \mathrm{mV}$ or more negative) (Tanaka et al., 2003). However, in the present study, direct measurement of $I_{\mathrm{h}}$ and calcium currents showed that those currents are either minimal or absent during the interspike interval of a template burst and hence do not directly contribute to maintaining spike discharge. Moreover, application of the specific $I_{\mathrm{h}}$ blocker ZD 7288 produced a very small outward current during the ISI that was attributed in subthalamic neurons to nonspecific block of outward $\mathrm{K}^{+}$currents (Do and Bean, 2003). It is not surprising that detectable inward $I_{\mathrm{h}}$ current was not observed in the present study, because the peak of the AHP template occurred at $-75 \mathrm{mV}$ where $I_{\mathrm{h}}$ shows minimal steady-state activation (Tanaka et al., 2003).

Although calcium current was not observed during the ISI, compared with the large $I_{\mathrm{NaT}}$, a smaller calcium current was observed during the action potential. This most likely is responsible for activation of calcium-dependent $\mathrm{K}^{+}$currents present in these neurons (Del Negro and Chandler, 1997), which do participate in control of burst duration but have minimal effects on spike frequency (Wu et al., 2001).
A surprising finding that may partially contribute to the highfrequency discharge observed in many Mes V neurons was the presence of $I_{\mathrm{NaR}}$. Similar to that elegantly described previously (Raman and Bean, 1997; Do and Bean, 2003), this current activates after spike repolarization from depolarized membrane potentials, and is maximally activated after repolarization to around $-40 \mathrm{mV}$ with a time constant of $<6 \mathrm{~ms}$ and voltage-dependent time constants of decay of $<30 \mathrm{~ms}$. Because Mes V neurons often discharge at frequencies $>90 \mathrm{~Hz}$, this current is well suited to provide a small "boosting" inward current immediately after spike repolarization and during the early part of the ISI trajectory when $I_{\mathrm{NaP}}$ is minimal. It is unlikely that the total inward sodium current flowing at the peak of the AHP trough is a classical "tail current," because the amplitude and time course of the total sodium current overlapped appreciably with the current flowing during this time and shortly thereafter when preceded by a depolarizing prepulse step voltage command that completely inactivated $I_{\mathrm{NaT}}$ (Fig. 5C).

Previously, we demonstrated that the membrane resonance observed in Mes V neurons results from the interaction of a lowthreshold, noninactivating $\mathrm{K}^{+}$current with the passive membrane RC network (Wu et al., 2001, 2005). We proposed that $I_{\mathrm{NaP}}$ amplifies the resonance and gives rise to subthreshold oscillations and burst generation (Wu et al., 2001, 2005). The present study directly demonstrates that during both subthreshold oscillations before burst discharge and during the ISIs within a burst, $I_{\mathrm{NaP}}$ is active and is the main contributor to inward current. Interestingly, after a spike, at the peak of the AHP trough $I_{\mathrm{NaP}}$ is not active (Fig. 4C), and this is likely attributable to its rapid deactivation kinetics and voltage dependence. Subsequently, $I_{\mathrm{NaP}}$ activates approximately one-third into the ISI and thereafter (Fig. 4C). Therefore, we would expect that this current significantly contributes to maintaining spike discharge, and reduction of this current would lead to burst termination. Consistent with this, block of $I_{\mathrm{NaP}}$ with riluzole suppresses subthreshold oscillations and burst discharge (Wu et al., 2005). Together, it is apparent that both $I_{\mathrm{NaR}}$ and $I_{\mathrm{NaP}}$ are important for maintaining spike discharge in Mes V neurons, whereas only $I_{\mathrm{NaP}}$ [which may include the window current component of $I_{\mathrm{NaT}}$ (Wu et al., 2005)] contributes to the onset of subthreshold oscillations before burst onset.

\section{Slow inactivation and burst termination}

Slow inactivation of sodium currents was demonstrated previously in a variety of CNS neurons and contributes to control of spike frequency and burst termination (Fleidervish and Gutnick, 1996; Pennartz et al., 1997; Magistretti and Alonso, 1999; Do and Bean, 2003). The present study showed that all three sodium currents $\left(I_{\mathrm{NaT}}, I_{\mathrm{NaR}}\right.$, and $\left.I_{\mathrm{NaP}}\right)$ are susceptible to slow inactivation that develops over a time course of seconds. This is similar to that demonstrated previously in subthalamic neurons using comparable methods (Do and Bean, 2003). Considering that these currents are active at various times during Mes $\mathrm{V}$ burst generation, it is reasonable to expect that reduction in these sodium conductances would contribute, although modestly, with additional currents to burst termination. Interestingly, our previous modeling data indicate that as little as a $6 \%$ reduction in the persistent sodium conductance results in suppression of Mes V burst discharge (Wu et al., 2005). Apparently, small changes in the balance of inward and outward currents from all sources will alter the pattern of spike discharge significantly. In the present study, we found that $I_{\mathrm{NaP}}$ was the most susceptible current to slow inactivation over the course of an $8 \mathrm{~s}$ burst or during a preceding $20 \mathrm{~s}$ depolarizing conditioning pulse, and this was coincident with a 
small decrease in spike frequency (Figs. 7, 8). However, it is unlikely that slow inactivation of $I_{\mathrm{NaT}}$ contributes substantially to burst termination because the safety factor for action potential initiation is large, although we have observed a progressive increase in spike threshold and decrease in spike amplitude during a burst (our unpublished observations). It is also unlikely that this decrease in amplitude during a burst can be fully explained by the small $(\sim 20 \%)$ decrease in sodium current observed in our study (Madeja, 2000) and, therefore, suggests that additional conductances, such as a calcium-dependent potassium conductance previously demonstrated in Mes V neurons (Del Negro et al., 1997; Wu et al., 2001), also contribute to spike amplitude reduction and burst termination. Regardless of the exact mechanism(s) responsible for burst termination, the shift in favor of a net outward current at burst termination must be small, because the resulting hyperpolarization is usually $\sim 3 \mathrm{mV}$ at most (Figs. 1, 9).

Slow inactivation during a burst and recovery from slow inactivation most likely is the mechanism for suppression of the subthreshold oscillations after burst termination and their subsequent emergence during the course of the interburst interval. After burst termination, a small, slow inward sodium current develops over the course of a few seconds that is associated with a parallel increase in amplitude of subthreshold oscillations. Superimposed on this slow inward current are rapid fluctuations in total membrane sodium current that are precisely correlated with the development of the rapid membrane potential oscillations. Although we did not measure the time course of recovery from inactivation, previously we found that $I_{\mathrm{NaP}}$ recovered from slow inactivation on the order of seconds (Wu et al., 2005); consistent with the time course of development of the inward sodium current and subthreshold oscillations during the interburst period. It is unlikely that the suppression of the subthreshold oscillations after burst termination results from a shift in voltage that is outside the activation for $I_{\mathrm{NaP}}$, because this conductance is maximally activated around $-45 \mathrm{mV}$, which is near the membrane potential at burst termination.

Clearly, sodium currents participate in multiple aspects of membrane excitability (Crill, 1996; Cantrell and Catterall, 2001) to shape neuronal discharge. Moreover, modification of these currents as a basis for certain pathophysiological conditions has now been established (Ashcroft, 2000). Interestingly, in DRG neurons, enhanced ectopic discharge occurring during nerve injury is associated with chronic pain and subthreshold oscillations (Amir et al., 1999). It remains to be seen whether any oral-motor disorders resulting in abnormal jaw movements and pain states are associated with dysfunction of sodium currents and subthreshold oscillations in brainstem neurons.

\section{References}

Alvarado-Mallart MR, Batini C, Buisseret-Delmas C, Corvisier J (1975) Trigeminal representations of the masticatory and extraocular proprioceptors as revealed by horseradish peroxidase retrograde transport. Exp Brain Res 23:167-179.

Amir R, Michaelis M, Devor M (1999) Membrane potential oscillations in dorsal root ganglion neurons: role in normal electrogenesis and neuropathic pain. J Neurosci 19:8589-8596.

Appenteng K, O’Donovan MJ, Taylor A (1978) Interneurones associated with the motor nucleus of the trigeminal nerve in the cat. J Physiol (Lond) 277:61P.

Appenteng K, Donga R, Williams RG (1985) Morphological and electrophysiological determination of the projections of jaw-elevator muscle spindle afferents in rats. J Physiol (Lond) 369:93-113.

Ashcroft FM (2000) Ion channels and disease. San Diego: Academic.

Baker R, Llinas R (1971) Electrotonic coupling between neurones in the rat mesencephalic nucleus. J Physiol (Lond) 212:45-63.
Beurrier C, Bioulac B, Hammond C (2000) Slowly inactivating sodium current $\left(I_{\mathrm{NaP}}\right)$ underlies single-spike activity in rat subthalamic neurons. J Neurophysiol 83:1951-1957.

Boehmer G, Greffrath W, Martin E, Hermann S (2000) Subthreshold oscillation of the membrane potential in magnocellular neurones of the rat supraoptic nucleus. J Physiol (Lond) 526:115-128.

Cantrell AR, Catterall WA (2001) Neuromodulation of $\mathrm{Na}^{+}$channels: an unexpected form of cellular plasticity. Nat Rev Neurosci 2:397-407.

Chandler SH, Hsaio CF, Inoue T, Goldberg LJ (1994) Electrophysiological properties of guinea pig trigeminal motoneurons recorded in vitro. J Neurophysiol 71:129-145.

Corbin KB, Harrison F (1940) Function of mesencephalic root of fifth cranial nerve. J Neurophysiol 3:424-435.

Crill WE (1996) Persistent sodium current in mammalian central neurons. Annu Rev Physiol 58:349-362.

Del Negro CA, Chandler SH (1997) Physiological and theoretical analysis of $\mathrm{K}^{+}$currents controlling discharge in neonatal rat mesencephalic trigeminal neurons. J Neurophysiol 77:537-553.

Dessem D, Taylor A (1989) Morphology of jaw-muscle spindle afferents in the rat. J Comp Neurol 282:389-403.

Do MT, Bean BP (2003) Subthreshold sodium currents and pacemaking of subthalamic neurons: modulation by slow inactivation. Neuron 39:109-120.

Enomoto A, Hsiao CF, Chandler SH (2004) Subthreshold and resurgent sodium currents in burst generation in mesencephalic trigeminal neurons. Soc Neurosci Abstr 30:397.10.

Fleidervish IA, Gutnick MJ (1996) Kinetics of slow inactivation of persistent sodium current in layer $\mathrm{V}$ neurons of mouse neocortical slices. J Neurophysiol 76:2125-2130.

Henderson G, Pepper CM, Shefner SA (1982) Electrophysiological properties of neurons contained in the locus coeruleus and mesencephalic nucleus of the trigeminal nerve in vitro. Exp Brain Res 45:29-37.

Hsiao CF, Del Negro CA, Trueblood PR, Chandler SH (1998) Ionic basis for serotonin-induced bistable membrane properties in guinea pig trigeminal motoneurons. J Neurophysiol 79:2847-2856.

Jackson AC, Yao GL, Bean BP (2004) Mechanism of spontaneous firing in dorsomedial suprachiasmatic nucleus neurons. J Neurosci 24:7985-7998.

Jerge CR (1963) Organization and function of the trigeminal mensencephalic nucleus. J Neurophysiol 26:379-392.

Khakh BS, Henderson G (1998) Hyperpolarization-activated cationic currents $\left(I_{\mathrm{h}}\right)$ in neurones of the trigeminal mesencephalic nucleus of the rat. J Physiol (Lond) 510:695-704.

Kolta A, Lund JP, Westberg KG, Clavelou P (1995) Do muscle-spindle afferents act as interneurons during mastication? Trends Neurosci 18:441.

Llinas R, Sugimori M, Simon SM (1982) Transmission by presynaptic spike-like depolarization in the squid giant synapse. Proc Natl Acad Sci USA 79:2415-2419.

Luo P, Dessem D (1996) Morphological evidence for recurrent jaw-muscle spindle afferent feedback within the mesencephalic trigeminal nucleus. Brain Res 710:260-264.

Madeja M (2000) Do neurons have a reserve of sodium channels for the generation of action potentials? A study on acutely isolated CAl neurons from the guinea-pig hippocampus. Eur J Neurosci 12:1-7.

Magistretti J, Alonso A (1999) Slow voltage-dependent inactivation of a sustained sodium current in stellate cells of rat entorhinal cortex layer II. Ann NY Acad Sci 868:84-87.

Miles TS (1979) Features peculiar to the trigeminal innervation. Can J Neurol Sci 6:95-103.

Morimoto T, Inoue T, Masuda Y, Nagashima T (1989) Sensory components facilitating jaw-closing muscle activities in the rabbit. Exp Brain Res 76:424-440.

Pape HC (1996) Queer current and pacemaker: the hyperpolarizationactivated cation current in neurons. Annu Rev Physiol 58:299-327.

Pedroarena CM, Pose IE, Yamuy J, Chase MH, Morales FR (1999) Oscillatory membrane potential activity in the soma of a primary afferent neuron. J Neurophysiol 82:1465-1476.

Pennartz CM, Bierlaagh MA, Geurtsen AM (1997) Cellular mechanisms underlying spontaneous firing in rat suprachiasmatic nucleus: involvement of a slowly inactivating component of sodium current. J Neurophysiol 78:1811-1825. 
Raman IM, Bean BP (1997) Resurgent sodium current and action potential formation in dissociated cerebellar Purkinje neurons. J Neurosci 17:4517-4526.

Raman IM, Bean BP (1999) Ionic currents underlying spontaneous action potentials in isolated cerebellar Purkinje neurons. J Neurosci 19:1663-1674.

Schurr A, West CA, Rigor BM (1988) Lactate-supported synaptic function in the rat hippocampal slice preparation. Science 240:1326-1328.

Stuart GJ, Dodt HU, Sakmann B (1993) Patch-clamp recordings from the soma and dendrites of neurons in brain slices using infrared video microscopy. Pflügers Arch 423:511-518.

Swensen AM, Bean BP (2003) Ionic mechanisms of burst firing in dissociated Purkinje neurons. J Neurosci 23:9650-9663.

Taddese A, Bean BP (2002) Subthreshold sodium current from rapidly inactivating sodium channels drives spontaneous firing of tuberomammillary neurons. Neuron 33:587-600.
Tanaka S, Wu N, Hsaio CF, Turman Jr J, Chandler SH (2003) Development of inward rectification and control of membrane excitability in mesencephalic v neurons. J Neurophysiol 89:1288-1298.

Verdier D, Lund JP, Kolta A (2004) Synaptic inputs to trigeminal primary afferent neurons cause firing and modulate intrinsic oscillatory activity. J Neurophysiol 92:2444-2455.

Wu N, Hsiao CF, Chandler SH (2001) Membrane resonance and subthreshold membrane oscillations in mesencephalic $\mathrm{V}$ neurons: participants in burst generation. J Neurosci 21:3729-3739.

Wu N, Enomoto A, Tanaka S, Hsiao CF, Nykamp DQ, Izhikevich E, Chandler SH (2005) Persistent sodium currents in mesencephalic v neurons participate in burst generation and control of membrane excitability. J Neurophysiol 93:2710-2722.

Zhang L, Krnjevic K (1993) Whole-cell recording of anoxic effects on hippocampal neurons in slices. J Neurophysiol 69:118-127. 